\title{
Percepciones socioculturales del paisaje simbólico: el Centro Histórico de Zacatecas, México
}

\author{
Ana Laura Santibáñez Coronado ${ }^{1}$ | Rubén Cantú Chapa ${ }^{2}$ | Gustavo Perez-Verdin ${ }^{3}$ \\ Recibido: 17-03-2020 | en su versión final: 28-01-2021
}

Resumen

\begin{abstract}
La importancia de conservar el paisaje reside en su propia definición que corresponde a la relación que existe entre humanidad-medio, una dualidad completamente vinculada, y que cada paisaje es diferenciable por sus propias características. El objetivo de la investigación es analizar la interpretación de los valores paisajísticos a través de la percepción social y cultural, además de reconocer sus componentes paisajísticos naturales y culturales del objeto de estudio: $\mathrm{El}$ Centro Histórico de Zacatecas, cuyo espacio expresa una herencia patrimonial importante. Metodológicamente a través de la comparativa entre las fotografías y encuestas aplicadas a cuatro sectores de la población: visitantes, habitantes, comerciantes y funcionarios públicos, se determinaron los componentes del paisaje más representativos para salvaguardar. Este método de recolección de datos estuvo integrado por cuatro etapas (1.- Caracterización, 2.- Diseño de encuesta, 3.- Resultados y 4.- Exposición de los hallazgos más significativos). A través de los resultados obtenidos, se reconocieron los significados simbólicos del paisaje de este centro histórico y se determinó que los componentes del paisaje representativos poseen características paisajísticas específicas que le asignan una imagen propia a la ciudad. La aportación de la investigación ofrece una propuesta de modelo de análisis para territorios con características similares a las del Centro Histórico de Zacatecas.
\end{abstract}

Palabras clave: Paisaje simbólico; percepción; centro histórico; modelo de análisis

Citación Histórico de Zacatecas, México, ACE: Architecture, City and Environment, 16(46), 9250. DOI: http://dx.doi.org/10.5821/ace.16.46.9250

\section{Sociocultural Perceptions of the Symbolic Landscape: Zacatecas Historical Center, México}

Abstract

\begin{abstract}
The importance to preserve the landscape lies in its own definition, which corresponds to the relationship that exists between humanity and the environment, a completely linked duality, and each landscape is distinguishable by its own characteristics. The objective of the research is to analyze the interpretation of landscape values through the social and cultural perception, in addition to recognizing its natural and cultural landscape components in the object of study: The Historic Center of Zacatecas, a site that expresses an important heritage. Methodologically, through the comparison between the photographs and surveys applied to four sectors of the population: visitors, inhabitants, merchants, and public officials, the most representative components of the landscape to safeguard were determined. This data collection method consisted of four stages (1. Characterization, 2. Survey design, 3. Results, and 4. Presentation of the most significant findings). Through the results obtained, the symbolic meanings of the landscape of this territory were recognized, and it was determined that the representative landscape components have specific characteristics that assign an image of their own in the city. The contribution of the research offers a proposal for an analysis model for territories with similar characteristics to those of this historic center.
\end{abstract}

Keywords: Symbolic landscape; perception; historic center; analysis model

1 Doctora en Ciencias en Conservación del Patrimonio Paisajístico, Instituto Politécnico Nacional-CIIEMAD (ORCiD: 0000-0002-2080-575X), ${ }^{2}$ Profesor investigador del CIIEMAD-IPN (ORCiD: 0000-0002-0104-3673), ${ }^{3}$ Profesor investigador del CIIDIR-IPN, Unidad Durango (ORCiD: 0000-0001-6788-2763). Correo de contacto: sc.analaura@gmail.com 


\section{Introducción}

Los retos del desarrollo urbano son muy diversos ya que se relacionan tanto con el crecimiento poblacional descontrolado, como con problemas económicos y socioambientales. Lo que concierne a la salvaguarda del paisaje, será pertinente definirlo como el territorio que vislumbra su propia complejidad, pues hace referencia al conjunto de elementos que diferencian y delimitan un sitio en particular. Es también parte esencial de nosotros y está íntimamente ligado a nuestra tradición, cultura y memoria histórica. El Centro Histórico de Zacatecas es un espacio que ofrece una herencia patrimonial y paisajística significativa, tanto para las zonas conurbadas como para el resto del país. Desde su fundación como ciudad minera en 1546 su disposición urbana se estableció en la proximidad de la cañada del Arroyo de la Plata que sirvió como su vía principal, además, acogió un trazado de "plato roto" en una topografía irregular propia de ciudades mineras. Este trazo urbano, a diferencia de otras ciudades novohispanas, no depende de una plaza central. Posee otras características del paisaje que bien vale subrayar como son los materiales constructivos empleados en su arquitectura: cantera rosa, herrería y madera (Cortés, 2014).

Este Centro Histórico ha sido escenario de luchas sociales específicas, por ejemplo, fue un punto estratégico para la culminación de la Revolución Mexicana; en esta ciudad aconteció la "Batalla de Zacatecas" el 23 de junio de 1914, que conllevó a la victoria del general Francisco Villa contra las fuerzas armadas del presidente Victoriano Huerta. En esta época, también, se le reconoció como un centro ferrocarrilero y dinamitero importante (Flores, de Vega, Kuntz y del Alizal, 2011).

Para el año de 1993 hubo un interés internacional para reconocerlo Patrimonio Cultural de la Humanidad por la UNESCO, dando apertura a la creación de planes y programas específicos para su salvaguarda. En la actualidad conserva su traza urbana original y es considerada una de las ciudades mineras mejor preservadas del mundo. Cabe destacar que, para el caso del Centro Histórico de Zacatecas, la UNESCO consideró que su valor correspondía al de "bien cultural" y pertenecía a los criterios II y IV, el primero corresponde a las obras maestras de la creación humana y el segundo a los sitios asociados directamente con los eventos o tradiciones vivas, con ideas o creencias, obras artísticas y literarias de notable importancia universal (UNESCO, 2004). Citando directamente los documentos presentados en la inclusión de la UNESCO de 1993 se subraya que:

CRITERIO II Zacatecas fue uno de los principales centros de la minería de plata desde el período colonial hasta el Siglo XX, su arquitectura y diseño reflejan la importancia económica y cultural que influyó en el crecimiento de estos sitios en el centro de México y América del norte. CRITERIO IV Zacatecas es un excelente ejemplo de un asentamiento colonial europeo que se adapta perfectamente a las restricciones impuestas por la topografía de su región montañosa y metalífera. (UNESCO, 1993, p. 34)

De acuerdo con su instrumento de planeación urbana más importante, la extensión de valor patrimonial del centro histórico constituye aproximadamente 441.34 hectáreas que contiene los monumentos arquitectónicos de mayor importancia de la ciudad (Gobierno el Estado de Zacatecas, 2016). El Estado de Zacatecas está ubicado en el noroeste del país (Ver Figura 1).

Según la Monografía geológico-minera del estado de Zacatecas (Cárdenas Cruz y Parga Pérez, 1991), su composición geológica está conformada principalmente de rocas sedimentarias, volcánicas mesozoicas y plutónicas cenozoicas. Es una entidad predominantemente argentífera con importantes yacimientos de plomo, zinc, cobre y, en menor proporción, oro. Así surgieron las actividades económicas de carácter minero que finalmente impulsaron el auge de la ciudad virreinal (Gobierno el Estado de Zacatecas, 2016).

ACE, 16 (4.6) CC BY-ND 3.0 ES | UPC Barcelona, España | Percepciones socioculturales del paisaje simbólico: el Centro Histórico de Zacatecas, México. DOI: http://dx.doi.org/10.5821/ace.16.46.9250 
Figura 1. Localización del Centro Histórico de Zacatecas

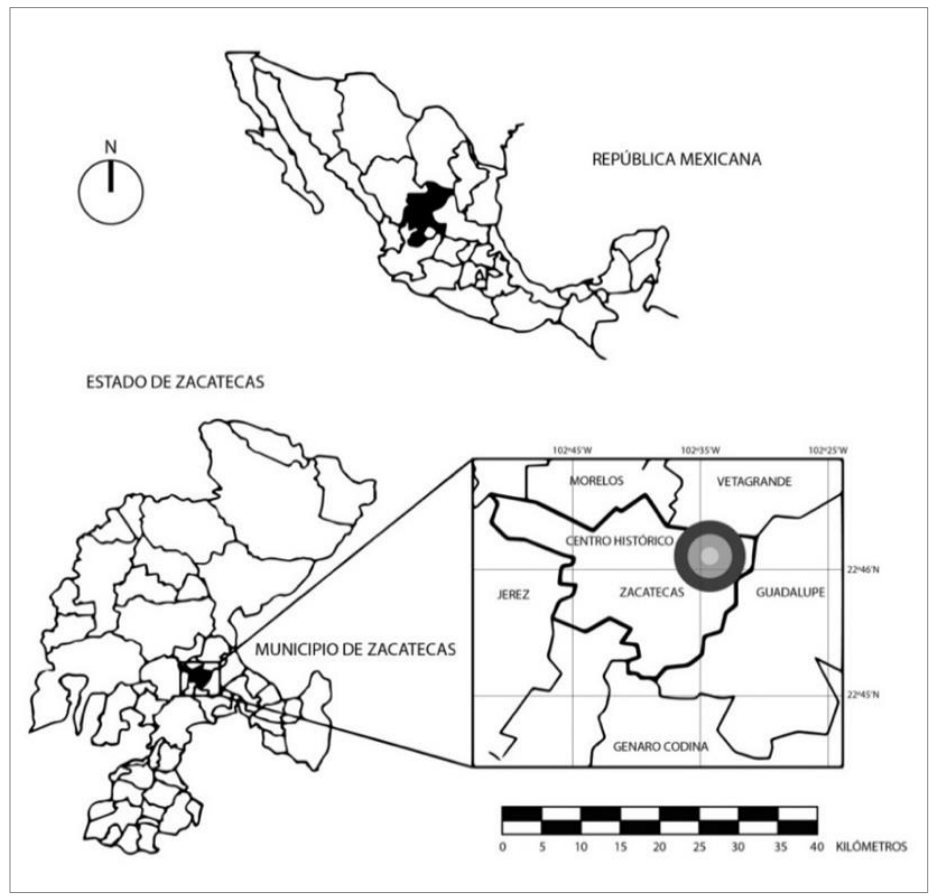

Fuente: Elaboración propia con base en información del Instituto Nacional de Estadística y Geografía (INEGI, 2020).

Esta investigación contempla el estudio de las percepciones socioculturales del paisaje del Centro Histórico de Zacatecas y el reconocimiento de sus valores y componentes a salvaguardar. El objetivo de la investigación fue el reconocer estos componentes paisajísticos -naturales y culturales- a través de la percepción social y cultural que, con su conjunto de variables, influyeron para su valoración histórica y reconocimiento por parte de los encuestados, y de los significados simbólicos que les atribuyeron.

Este artículo contiene el análisis del área de estudio, sus antecedentes históricos relevantes, las características geográficas, el reconocimiento de los valores y componentes socioculturales del paisaje. Los resultados respondieron a la pregunta de investigación central, ¿Es el paisaje la representación de la imagen percibida que ha trascendido y es vital para satisfacer las necesidades simbólicas actuales y futuras? La contribución de la investigación reside en mostrar la importancia de la conservación paisajística del Centro Histórico de Zacatecas en la representatividad de su propio paisaje.

\section{Marco teórico conceptual}

Para esta investigación, la conceptualización del paisaje corresponde a la relación que existe entre humanidad-medio, una dualidad completamente vinculada y cada paisaje es diferenciable por sus propias características. Pensar el paisaje como un producto social que resulta de la evolución colectiva, además de ser el reflejo de su organización que responde a sus propias complejidades. Autoras como Manzini (2017), definen también que los paisajes son dinámicos y se transforman en el tiempo, pero habrá que comprender su significado histórico para el entendimiento de sus valores. Por otro lado, Robles (2017), sugiere que cada paisaje posee sus propias características y dinámicas particulares al ser estos híbridos por adaptarse a las diversas necesidades, valores, actividades y significados de quienes los habitan.

ACE, 16 (4.6) CC BY-ND 3.0 ES | UPC Barcelona, España | Percepciones socioculturales del paisaje simbólico: el 3 Centro Histórico de Zacatecas, México. DOI: http://dx.doi.org/10.5821/ace.16.46.9250 
Para sustentar la idea inicial sobre la relación humanidad-medio, que es tema central de la investigación, autores como Nogué (2017) reflexionan sobre el ser humano como habitante de un territorio en particular, este distingue el paisaje que desea ver, es decir, el paisaje es una idea construida socialmente que surge de su apropiación y apreciación territorial. Así las percepciones del paisaje son tan diversas y complejas que van evolucionando a través del tiempo, desarrollándose de esta manera nuevas nociones. El paisaje del Centro Histórico de Zacatecas es el sitio donde se revelan todas las percepciones de cada uno de sus actores y los componentes paisajísticos- culturales y naturales- son la vía para asignarle su valor simbólico. La importancia de conservar estos componentes reside en su valor patrimonial, porque forman parte de su identidad y de la construcción de la memoria colectiva, además de ser la clave para su desarrollo humano sostenible.

La teoría de la percepción fue una noción clave para esta investigación, de la cual han surgido diversos estudios que vale la pena mencionar. En Alemania, el movimiento de la Gestalt del siglo XX liderado por Wertheimer, Koffka y Köhler, consideró a la percepción como un proceso esencial de la actividad mental de la cual dependen el aprendizaje, la memoria y el pensamiento (Oviedo, 2004). Además, la Teoría de la Gestalt afirma que la percepción es un proceso mental de extracción de la información que supone es la más importante para cada individuo, para lograr mayor grado de racionalidad y coherencia del medio que los rodea.

Por otro lado, el modelo de acción razonada propuesto por Ajzen y Fishbein (citado en Stefani, 2005, p. 22-23) entiende que el comportamiento social reacciona a los actos de la voluntad, es decir, que un individuo racional realiza una acción o adopta una conducta específica de acuerdo con la información que dispone, ya sea de manera normada o sistematizada. Así, la percepción del paisaje está relacionada con el modelo de acción razonada al responder a las conductas sociales, creencias y acciones específicas. También, se ha demostrado que existen diversas variables que influyen en la percepción y que tienen que ver con nuestras experiencias personales, edad, entorno, profesión e inclusive el género. Para reforzar lo anterior, autores como Edney (1976), quien comprobó los efectos que tiene la experiencia previa del entorno con el comportamiento, por ejemplo, la percepción del tamaño de un lugar cambia significativamente si se conoce con anterioridad. De manera similar, el experimento de Smith (1984) reveló que en cuanto más fuera reconocido un monumento o sitio del caso de estudio, mejor lo evaluaron los encuestados al asignarles los valores de: agradable o significativo. También otro hallazgo importante del experimento fue que pudieron ubicar estos sitios con mayor precisión en un mapa.

Para Holl (2018) la percepción personal depende de la superposición de perspectivas urbanas que están determinadas por nuestros trayectos y movimientos. Ninguna vista única de un elemento urbano será completada sin "múltiples perspectivas" y con el reconocimiento de vías alternativas de desplazamiento por las ciudades que habitamos (Holl, 2018, p. 20-21). Desde el estudio local de la percepción para habitantes de la Reserva de la Biosfera en tres zonas de La Michilía, al sur del estado de Durango en México de Perez-Verdin et al. (2004), las percepciones fueron la base para crear las estrategias de participación de sus residentes en foros públicos relacionados con dinámicas al aire libre y la planificación de la recreación en esta zona. Además, con este análisis se detectaron los impactos potenciales de mayor riesgo de estas actividades como son los incendios forestales, el hacinamiento y la pérdida de valores tradicionales, sólo por mencionar algunos. Para este estudio la variable del entorno y de las experiencias personales de los encuestados influyeron en su percepción.

El simbolismo del paisaje que es nuestro concepto central, algunos autores distinguen los principales criterios perceptivos: la escala, los esquemas lógicos y las referencias (Kjellberg 1972 y Leroy, 1971, citados en Bailly, 1978, p. 51-56); cabe acentuar en los últimos criterios, al señalar que el sujeto utiliza los puntos de referencia para familiarizarse con su ciudad y sentirla, ya sean el relieve como los puntos geográficos o/y los simbólicos como sus monumentos. Desde este enfoque, Pallasmaa (2018)

ACE, 16 (4.6) CC BY-ND 3.0 ES | UPC Barcelona, España | Percepciones socioculturales del paisaje simbólico: el 4 Centro Histórico de Zacatecas, México. DOI: http://dx.doi.org/10.5821/ace.16.46.9250 
evidencia el papel de la arquitectura como la articuladora de nuestras experiencias en el mundo y fortalece nuestra realidad. Lo significativo de los edificios se encuentra más allá de la propia arquitectura, está relacionado con nuestro propio conocimiento y experiencia corporal-espiritual de nuestro entorno (Pallasmaa, 2018, p. 13).

Por otro lado, Vergara (2006) menciona que la imagen de la ciudad es la imagen viva que se crea y recrea permanentemente en la cotidianidad de sus habitantes, además de indicar que esta imagen surge de la ciudad material que hemos ido construyendo a través de generaciones, sobre nuestros intereses y sueños. Así deducimos que el simbolismo del paisaje lo erige la propia población.

También cabe resaltar que para Bailly (1978), quien realiza una interesante reflexión, al referir que "son numerosas las personas que eligen idénticos criterios de identificación de los sectores urbanos. Se trata de una forma de imagen colectiva ligada a la historia, la cultura y los vínculos simbólicos; tal como la que los individuos tejen con determinados barrios" (Bailly ,1978, p. 113).

En conclusión, el estudio del Centro Histórico Zacatecas a través de la imagen colectiva de los encuestados se erigieron los vínculos simbólicos con los componentes del paisaje (culturales y naturales) más significativos de acuerdo con su propia percepción, es decir, los propios sectores poblacionales asignaron los componentes simbólicos del paisaje. Las variables socioculturales que influyeron en la percepción del paisaje del Centro Histórico de Zacatecas tuvieron que ver con el reconocimiento de la historia, tipos de actividades -sectores poblacionales-, valoración del patrimonio y datos demográficos -edad, profesión, ingresos, etc.- este análisis se diferencia de otras investigaciones al integrar las percepciones de todos sus actores y de los significados simbólicos que les atribuyeron.

Figura 2. Análisis de la percepción sociocultural del caso de estudio

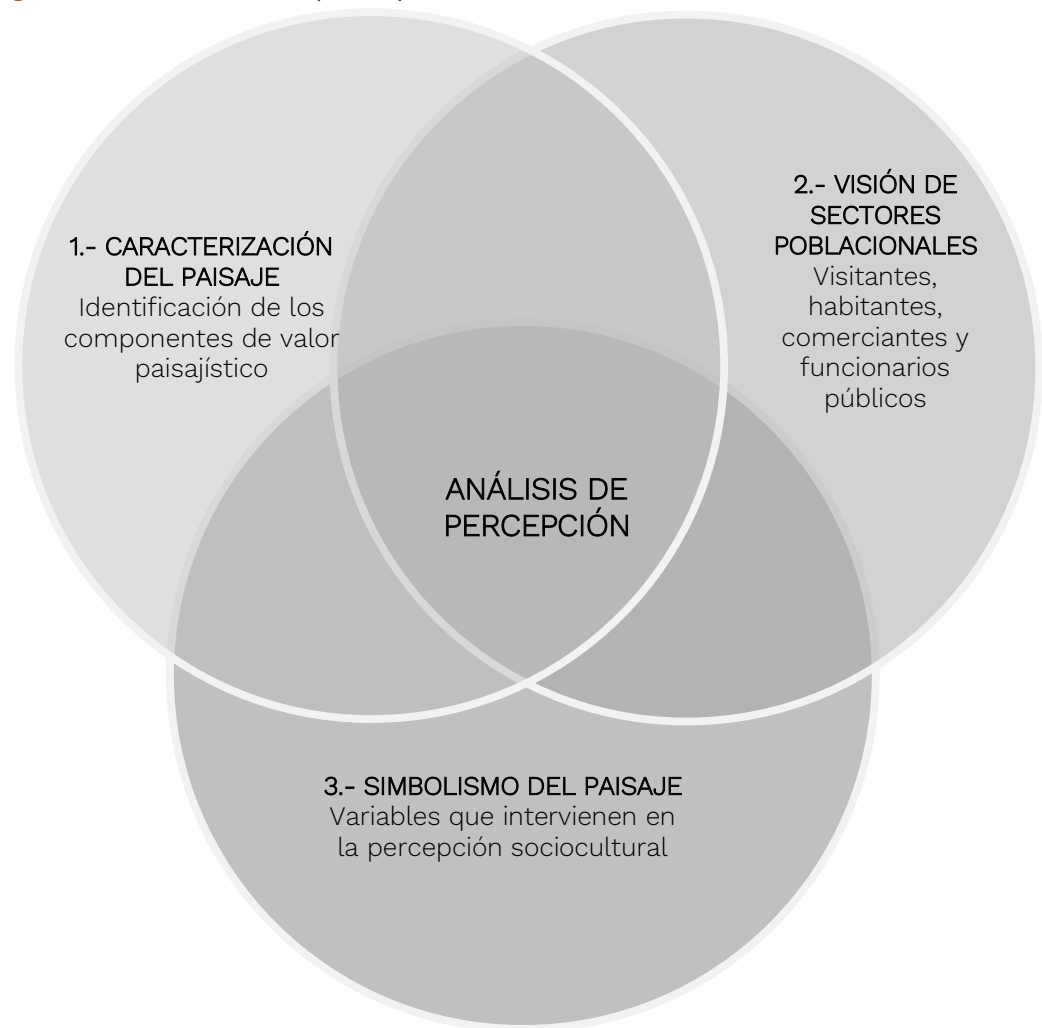

Fuente: Elaboración propia con base en el marco teórico desarrollado.

ACE, 16 (4.6) CC BY-ND 3.0 ES | UPC Barcelona, España | Percepciones socioculturales del paisaje simbólico: el Centro Histórico de Zacatecas, México. DOI: http://dx.doi.org/10.5821/ace.16.46.9250 
Finalmente, y de acuerdo con el marco teórico desarrollado, el análisis de la percepción sociocultural del Centro Histórico de Zacatecas estuvo encaminado hacia tres pilares: 1.-la identificación de los componentes del paisaje, 2.- la visión de cada sector, 3.- las variables que intervinieron en la percepción y su significado simbólico (Ver Figura 2). En la parte conclusiva se describe la aportación de la investigación al mostrarse la importancia de la conservación paisajística del centro histórico y en la representatividad simbólica para las generaciones futuras y actuales.

\section{Análisis y Recolección de Datos}

La obtención de datos para este estudio consistió en cuatro etapas: 1) Caracterización; 2) Diseño de encuesta; 3) Los resultados a partir del tamaño de muestras; y finalmente 4) Exponer los hallazgos más significativos de la percepción del paisaje simbólico del Centro Histórico de Zacatecas.

\subsection{Etapa de Caracterización}

En esta fase se analizaron e identificaron los componentes culturales y naturales que conforman el paisaje del Centro Histórico de Zacatecas, que corresponden a sitios con características homogéneas y rasgos propios distintivos para demarcar un hito y son reconocibles en el paisaje por sus particularidades. Además, se identificaron y describieron cada uno de ellos, aunque este sitio posee vastos componentes significativos, conforme a los resultados obtenidos en las encuestas, se seleccionaron los diez más reconocibles: seis componentes culturales y cuatro naturales (Ver Figura 3 y Tabla 1).

Figura 3. Collage de los componentes paisajísticos cultuales y naturales representativos del Centro Histórico de Zacatecas

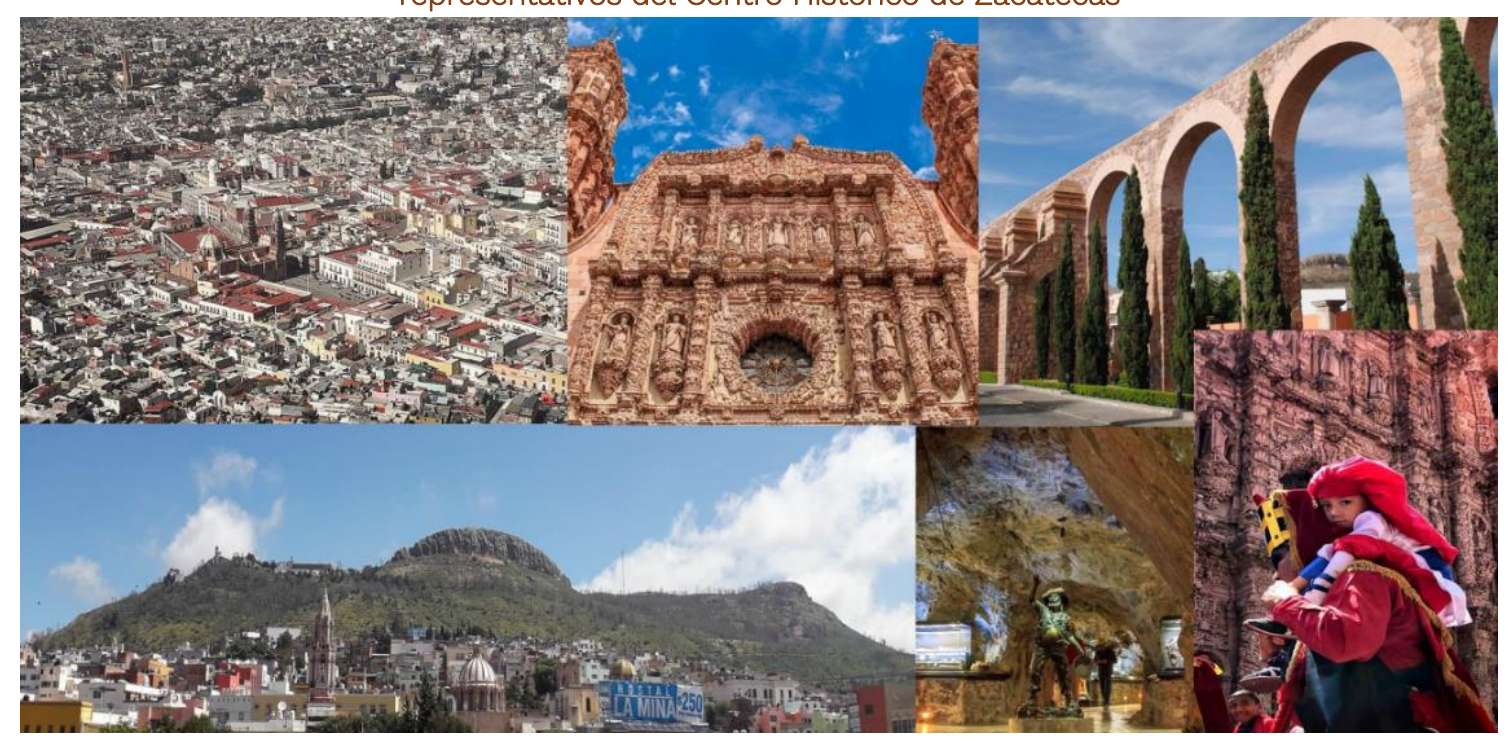

Fuente: Elaboración propia con base en el trabajo de campo con fotografías propias y de los fotógrafos Rafael Medina Briones y Bernardo Candelas de la Torre.

Cabe destacar que estos componentes están integrados dentro los "Polígonos de Protección" que fueron delimitados de acuerdo con la declaratoria por la UNESCO y del "Proyecto de Declaratoria del Instituto Nacional de Antropología e Historia” del año 2012, que después fueron conciliados con la "Ley de Protección y Conservación del Patrimonio Cultural del Estado de Zacatecas" (Gobierno del Estado de Zacatecas, 2016) y corresponden a:

ACE, 16 (4.6) CC BY-ND 3.0 ES | UPC Barcelona, España | Percepciones socioculturales del paisaje simbólico: el 6 Centro Histórico de Zacatecas, México. DOI: http://dx.doi.org/10.5821/ace.16.46.9250 
- Zona A. Zona de conservación-protección del núcleo histórico patrimonial de la ciudad de Zacatecas y sus barrios.

- Zona B. Correspondiente a la "Zona de transición" que comprende las colonias que delimitan el núcleo histórico.

- Zona C. "Zona de entorno paisajístico" se ubican las áreas naturales que han sido referencias visuales y culturales (Ver Figura 4). Dentro de estos perímetros se pudieron identificar la localización de los hitos simbólicos del paisaje y se reveló que la Zona A contiene el mayor número de componentes culturales, mientras que la zona $\mathrm{C}$, los componentes naturales.

Tabla 1. Identificación de los componentes culturales y naturales del paisaje

\begin{tabular}{|l|l|l|l|l|l|}
\hline Componentes & Nombre & Valor & Categoría & Perímetro & Importancia Histórica \\
\hline \hline \multirow{5}{*}{ Culturales } & Catedral & Arquitectónico & Edificación & A & Relevante \\
\cline { 2 - 6 } & Acueducto el Cubo & Arquitectónico & Infraestructura & A & Poco relevante \\
\cline { 2 - 6 } & Arquitectura colonial & Arquitectónico & Edificación & A Y B & Relevante \\
\cline { 2 - 6 } & Colores y texturas & Urbano & Cultural & A, B Y C & Poco relevante \\
\cline { 2 - 6 } & Festividades & Social & Cultural & A, B Y C & Relevante \\
\cline { 2 - 6 } & Traza Urbana & Urbano & Infraestructura & A Y B & Relevante \\
\hline \multirow{5}{*}{ Naturales } & Cerro de la Bufa & Histórico & Geomorfológica & C & Relevante \\
\cline { 2 - 6 } & Mina el Edén & Histórico & Geomorfológica & C & Relevante \\
\cline { 2 - 6 } & Relieve & Imagen urbana & Geomorfológica & A, B Y C & Relevante \\
\cline { 2 - 6 } & Espacios públicos & Urbano & Infraestructura & A, B Y C & Poco Relevante \\
\hline
\end{tabular}

Fuente: Elaboración propia con base en los datos obtenidos en encuestas.

Figura 4. Perímetros de protección del Centro Histórico de Zacatecas

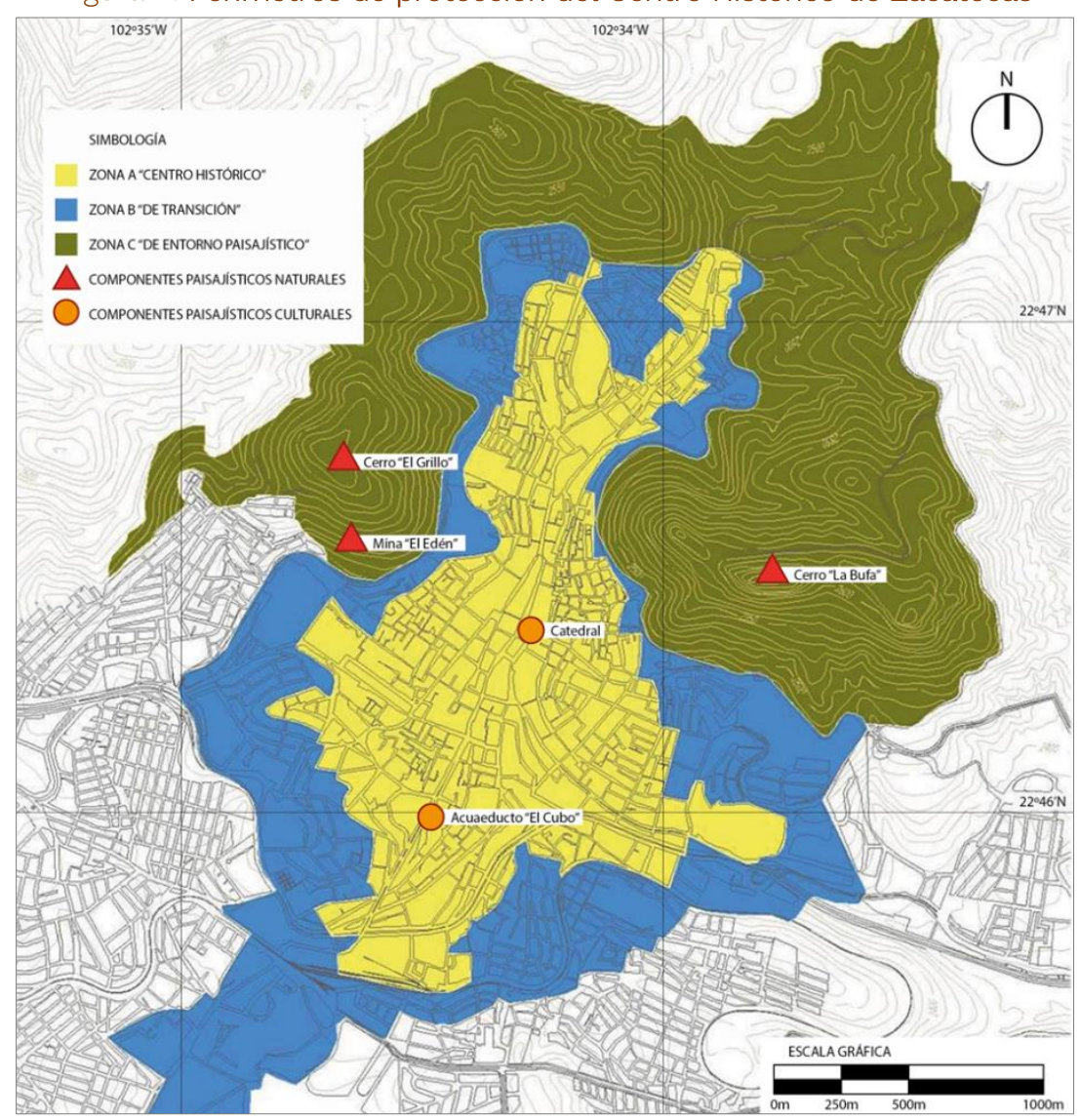

Fuente: Elaboración propia con información del Programa Parcial del Centro Histórico de Zacatecas 2016-2040.

ACE, 16 (4.6) CC BY-ND 3.0 ES | UPC Barcelona, España | Percepciones socioculturales del paisaje simbólico: el 


\subsubsection{Componentes culturales}

En los recorridos y visitas al centro histórico se definieron los componentes culturales como aquéllos que poseen características patrimoniales tangibles e intangibles excepcionales, sobre todo, monumentos, tipologías visuales reconocibles y a las expresiones artísticas -festividades tradicionales- que tienen lugar en el paisaje. Los destacables son:

Tangibles:

- La catedral. Su fachada corresponde al estilo barroco en el que destacan materiales y técnicas de construcción sobre la cantera color rosa labrada, típica de la región. Aunque su construcción inició en 1752, fue concluida en 1904 al terminar el remate y la torre norte (Flores et al., 2011). La portada principal, enmarcada sobre el eje vial más importante de la ciudad, es un elemento arquitectónico simbólico y de identidad paisajística.

- El acueducto "El Cubo" fue construido entre los siglos XVII y XIX. Consistió en la obra de infraestructura urbana más trascendente de la época ya que suministró por un largo periodo agua a la ciudad (Zárate, 2016). Aunque en la actualidad es poca la evidencia que se conserva del acueducto lo que persiste es reconocible para sus habitantes.

- Arquitectura colonial. El Centro Histórico de Zacatecas se destaca por sus monumentos históricos en los que predomina la cantera rosada, la herrería y la madera. La estricta reglamentación de imagen urbana ha propiciado la conservación de las fachadas antiguas, y limita los diseños de anuncios en los comercios, favoreciendo la preservación del paisaje histórico- urbano.

- Colores y texturas. La imagen del centro histórico está personificada por el matiz de sus construcciones coloniales en conjunto con el medio natural, son reconocidas por su singularidad tanto arquitectónica como por el uso de cantera de color rosa y material autóctono. Mantener la apariencia de la ciudad es una de las metas de la Junta de Protección y Conservación de Monumentos y Zonas Típicas del Estado de Zacatecas, quien ha elaborado un manual para orientar al público para la elección de colores en fachadas. Este manual retoma los criterios de la Ley de Protección y Conservación del Patrimonio Cultural del Estado de Zacatecas (Gobierno del Estado de Zacatecas, 2018), esto sin duda ha propiciado la homogenización de la imagen visual del paisaje y de su percepción, asignándole tonalidad de colores propios del lugar.

- Traza urbana. Los primeros asentamientos tuvieron que adecuarse a la topografía irregular de la región. La ciudad fue fundada el 8 de septiembre de 1546 y fue hasta 1573, por la promulgación de Leyes de Indias por Felipe II, que se legisla por decreto como Ciudad Real de trazado regular. La morfología del centro histórico es una de las características que destaca por el modo de adaptarse a su medio y por la apropiación del territorio.

Intangibles:

- Festividades. La identidad del centro histórico también está explícita por las actividades culturales y tradiciones que se celebran durante todo el año. El paisaje es el escenario de expresiones que han permanecido durante varias generaciones. "Las Morismas de Bracho", una festividad local que tiene lugar desde 1824 sobre el Cerro de San Martín, aledaño al centro histórico, y que contempla un desfile que pasa por su vialidad principal, es una de las representaciones más importantes y antiguas del estado y del país. Las "Morismas de Bracho" es una representación de la lucha entre dos bandos, el moro y el cristiano que se personifica cada año y a través de ella se narran las batallas de Felipe II, donde el paisaje del Centro Histórico de Zacatecas es el escenario protagónico de esta festividad.

El evento dura cuatro días y tres noches, en la que participan de entre ocho mil actores. La representación de estas batallas personifica la vivencia colectiva y la ritualidad de sus

ACE, 16 (4.6) CC BY-ND 3.0 ES | UPC Barcelona, España | Percepciones socioculturales del paisaje simbólico: el 
participantes, al simbolizar una tradición comunitaria que ha trascendido generaciones, ya que también se involucran familias completas en la escenificación (Vértiz y Alfaro, 2001). Las representaciones de moros y cristianos son muy extensas y antiguas, se reconocen sus inicios en la Edad Media y su mayor desarrollo en el modelo valenciano de la actual provincia de Alicante en España a partir del siglo XIX. Como fenómeno social y festivo podrá ser estudiado desde sus procesos históricos para comprender sus orígenes y su clasificación en todo el mundo (Domene Verdú, 2015). La tesis de García Sandoval (2014) sostiene que en Zacatecas esta festividad fue adoptada durante la época de la Colonia, cuando los conquistadores lograron establecer sus instituciones y la reproducción de sus costumbres e identidad al conjugar su fe y el servicio al rey. También describe que en los relatos del conquistador español Bernal Díaz del Castillo la primera representación de moros y cristianos se efectuó en territorios del continente americano en 1538 (García Sandoval, 2014, p. 54).

\subsubsection{Componentes naturales}

La enunciación de los componentes naturales del paisaje surgió del reconocimiento de los sitios con características simbólicas del medio natural y que tienen poca intervención humana.

- El Cerro de la Bufa. Esta elevación forma parte de la zona denominada de "entorno paisajístico" y es vital su conservación, ya que constituye un sitio de valor histórico y escénico. Desde la llegada del primer explorador español y, en su búsqueda de un posible sitio para la extracción de oro y plata, se reconoció la potencialidad el cerro por sus características naturales. También fue territorio clave para la culminación de la ya mencionada "Batalla de Zacatecas", entre otros sucesos importantes.

- $\quad$-Mina el Edén. Aunque no sea un componente meramente natural, se integra en este grupo ya que está inserta en una de las elevaciones de "El Cerro del Grillo". Esta mina fue detonante de la principal actividad económica y del primer asentamiento urbano de la ciudad. Actualmente es un sitio exclusivo para actividades turísticas, ya que dejaron de extraerse minerales en 1960 para evitar desplomes en el suelo urbano.

- $\quad$-Relieve. El conjunto de elevaciones han sido escenario de múltiples eventos e hitos del paisaje; para la memoria urbana son sitios permanentes de referencia histórica. Los cerros que delimitan el centro histórico que son representativos: el Cerro del Grillo y el Cerro de La Bufa.

- - Espacios Públicos. El centro histórico posee variedad de plazas y jardines que funcionan como sitios de encuentro social, de entretenimiento, además resguarda especies de árboles endémicas de la región. Los espacios públicos más recurrentes son la Alameda, la Plaza de Armas y el Jardín Juárez.

\subsection{Etapa del Diseño de la encuesta}

La investigación está sustentada en las preguntas cerradas de opción múltiple que se realizaron durante las encuestas levantadas. Fueron aplicadas individualmente para mostrar las escalas de la evaluación con varias categorías y se le asignó un valor a cada respuesta. A 'Muy importante' le correspondía el 3 y 'Poco importante' el 1, esta medición se utilizó, por ejemplo, para reconocer las tendencias de las preferencias y el reconocimiento del paisaje a través de la percepción de los encuestados. La ordenación y diseño de la encuesta consistió en 26 preguntas estructuradas a través de tres variables examinadas (Ver Figura 5).

1. Patrimonio y paisaje. Preguntas relacionadas sobre el reconocimiento del valor de preservar el patrimonio paisajístico y su reconocimiento.

ACE, 16 (4.6) CC BY-ND 3.0 ES | UPC Barcelona, España | Percepciones socioculturales del paisaje simbólico: el 
2. Reconocimiento de la historia. Las preguntas tuvieron que ver con el conocimiento de los eventos históricos y del grado de identificación con los valores paisajísticos por los encuestados.

3. Demografía. Preguntas con relación a los datos demográficos, tales como ciudad de residencia, ocupación, género, ingresos, edad y escolaridad.

Figura 5. Estructura de la encuesta

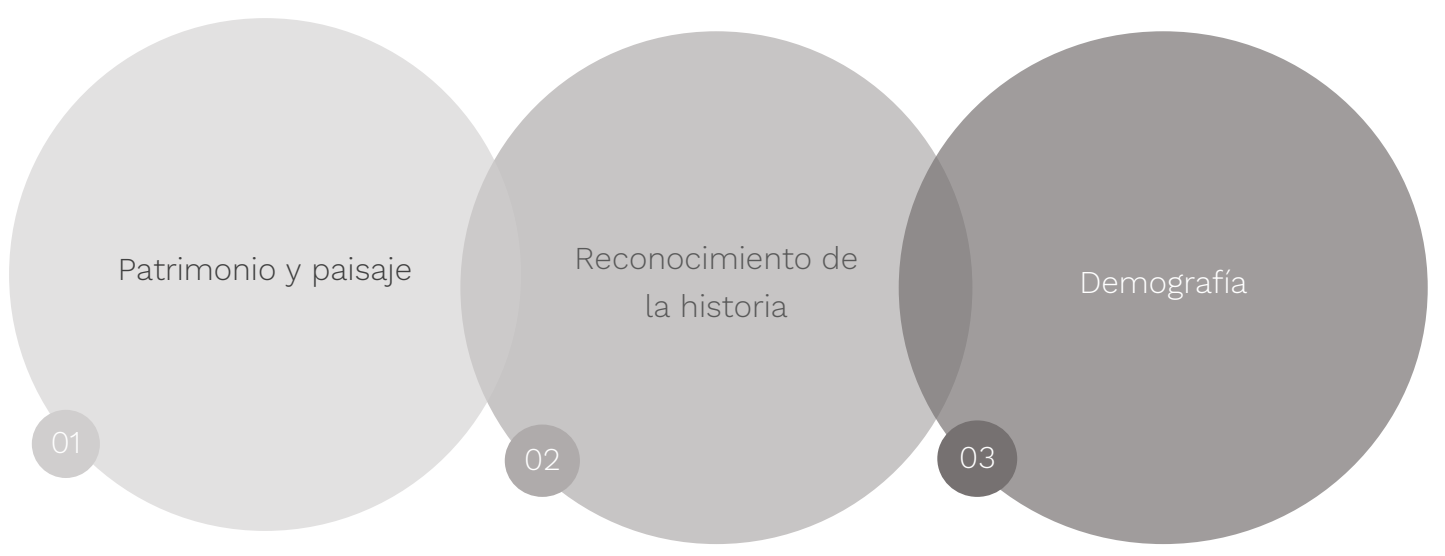

Fuente: Elaboración propia.

\subsection{Tamaño de la muestra}

Para establecer el número de encuestas aplicadas a los cuatro sectores de la población: visitantes, habitantes, comerciantes y funcionarios públicos, se utilizó la fórmula sugerida por Rea y Parker (2014). En este tipo de investigaciones se considera que el margen de error no debe exceder el 10\% -aunque es frecuente utilizar los rangos del 3\% al 5\%- (Rea y Parker, 2014, p. 167), sin embargo, para este estudio fue adecuado optar por el valor de 10\%, esto derivado de los resultados de una muestra piloto donde la variación entre la población no fue muy alta. Además, se obtuvieron estadísticas de fiabilidad de Alfa de Cronbach con valor de 0.988 a través del software SPSS, los cuales fueron consistentes por su proximidad al valor de 1. Para estimar el tamaño de muestra se consideraron los diferentes sectores involucrados.

El total de visitantes a museos registrados en el año 2016 -que es la última estimación tomada por el Gobierno del Estado- tiene un total de 209,015 personas durante ese año. ${ }^{1}$

Para el sector de los habitantes del Centro Histórico se utilizó, para estimar el tamaño de la muestra, los 15 AGEBS $^{2}$-Áreas Geoestadísticas Básicas- integrados al Perímetro A: núcleo histórico patrimonial, que consistió en 25,561 residentes. De acuerdo con los Censos Económicos INEGI (2014), el personal ocupado en el sector de comercio fue de 11,463 personas en el municipio de Zacatecas donde se encuentra el centro histórico.

Posteriormente, para el total de personal trabajando se registraron 163 individuos en las seis instituciones encargadas en la salvaguarda del patrimonio: Junta de Protección y Conservación de

\footnotetext{
${ }^{1}$ Fuente: INEGI- Anuario estadístico y geográfico de Zacatecas 2017. Datatur. La información corresponde a los museos administrados por el Instituto Zacatecano de Cultura "Ramón López Velarde" y comprende tanto a los visitantes con boleto pagado, como aquellos que corresponden a grupos de cortesía.

${ }^{2}$ Censo de Población y Vivienda 2010. INEGI.
}

ACE, 16 (4.6) CC BY-ND 3.0 ES | UPC Barcelona, España | Percepciones socioculturales del paisaje simbólico: el Centro Histórico de Zacatecas, México. DOI: http://dx.doi.org/10.5821/ace.16.46.9250 
Monumentos Históricos, Secretaría de Desarrollo Urbano y Vivienda y Ordenamiento Territorial, Instituto Regional del Patrimonio Mundial, Instituto Zacatecano de Cultura, INAH-Zacatecas y Secretaría de Turismo. En síntesis, el tamaño de la población total referente a los cuatro sectores de la población explorada en el Centro Histórico de Zacatecas fue de 246,202 personas de las cuales se estimó el siguiente tamaño de muestra (Ver Tabla 2):

Tabla 2. Tamaño de muestra por sector poblacional

\begin{tabular}{|r|l|r|r|}
\hline Tamaño población & \multicolumn{1}{|c|}{ Sector poblacional } & Porcentaje por tamaño poblacional & No. encuestas aplicadas \\
\hline 209.015 & 1. Visitantes & $84.90 \%$ & 85 \\
\hline 25.561 & 2. Habitantes & $10.38 \%$ & 10 \\
\hline 11.463 & 3. Comerciantes & $4.65 \%$ & 5 \\
\hline 163 & 4. Servidores públicos & $0.07 \%$ & 6 \\
\hline Total 246.202 & Cuatro sectores & Porcentaje Total: $100,00 \%$ & Total: 106 \\
\hline
\end{tabular}

Fuente: Elaboración propia.

Este tamaño de muestra se comprobó con la fórmula de Rea y Parker (2014), para poblaciones mayores de 100,000 personas. Así se determinó que debían aplicarse como mínimo 96 encuestas. Al final se incrementó la cifra al aplicar 106 en los cuatro sectores de la población mencionados anteriormente y se confirman los datos con la formula a continuación.

$$
\begin{gathered}
\mathrm{n} 1=\left\lfloor\left.\frac{\mathrm{Z} \alpha \sqrt{\mathrm{P}(1-\mathrm{P})}}{\mathrm{Cp}}\right|^{2}=\mathrm{n} 1=\left\lfloor\left.\frac{1.96 \sqrt{0.5(1-0.5)}}{0.1}\right|^{2}=96\right.\right. \\
\text { Donde: } \\
\text { Tamaño de población }=246,202 \\
\text { Nivel de confianza (95\%) } \mathrm{Z} \alpha=1.96 \\
\text { Margen de error } \mathrm{C} \mathrm{p}=0.10 \\
\text { Proporción } \mathrm{P}=0.50
\end{gathered}
$$

Fuente: Elaboración propia con base en Rea y Parker.

\section{Etapa de Resultados}

Esta fase del análisis consistió en reconocer, a través de la percepción, la importancia y el valor que fue asignado por cada sector de la población a los componentes culturales y naturales del paisaje. Posterior a este reconocimiento, se interpretaron las respuestas obtenidas y presentaron los componentes más reconocibles y los menos valorados por los encuestados (Ver Tabla 3). En general, el mayor porcentaje de los cuatro sectores de la población encuestados coincidieron en que el presupuesto asignado para la mejora del Centro Histórico de Zacatecas debería destinarse para la restauración de monumentos y limpieza de calles -visitantes 50.6\%, habitantes 60\%, comerciantes $40 \%$ y servidores públicos $66.7 \%$-. Aunque se realizan diversas actividades en el territorio, coinciden en que los instrumentos normativos deben enfocar sus estrategias a estas acciones.

De los componentes paisajísticos que deberían salvaguardarse, cada sector también coincidió en que la Catedral y el Cerro de la Bufa son los que poseen mejor valoración y deben ser heredados a generaciones futuras, por ser testigos de la historia- visitantes $68.25 \%$, habitantes 60\%, comerciantes $80 \%$ y servidores públicos $66 \%-$.

La población de mujeres encuestadas fue mayor al obtenerse los siguientes porcentajes: $54.7 \%$ mujeres y $45.3 \%$ hombres. La ocupación que predominó fue la de jubilado con un $27.3 \%$, a pesar de

ACE, 16 (4.6) CC BY-ND 3.0 ES | UPC Barcelona, España | Percepciones socioculturales del paisaje simbólico: el Centro Histórico de Zacatecas, México. DOI: http://dx.doi.org/10.5821/ace.16.46.9250 
que el rango de edad dominante fue de 20 a 40 años con un 44.3\%. En general, los cuatro sectores de la población han considerado que la valoración del patrimonio paisajístico reside en la conservación de los componentes más simbólicos.

Tabla 3. Orden de valoración de los componentes culturales y naturales

\begin{tabular}{|l|l|l|}
\hline Componentes & Nombre & Orden de reconocimiento \\
\hline \multirow{4}{*}{ Culturales } & Catedral & Muy simbólico \\
\cline { 2 - 3 } & Arquitectura colonial & Simbólico \\
\cline { 2 - 3 } & Acueducto el Cubo & Poco simbólico \\
\cline { 2 - 3 } & Festividades & Muy poco simbólico \\
\hline \multirow{4}{*}{ Naturales } & Cerro de la Bufa & Muy simbólico \\
\cline { 2 - 3 } & Mina el Edén & Simbólico \\
\cline { 2 - 3 } & Relieve & Poco simbólico \\
\cline { 2 - 3 } & Espacios públicos & Muy poco simbólico \\
\hline
\end{tabular}

Fuente: Elaboración propia con base en los datos obtenidos en encuestas.

De acuerdo con la interpretación de las respuestas obtenidas en las encuestas se determinó que a la Catedral se le designó como el componente cultural más reconocido y valorado. La mayoría de los encuestados coincidieron que es el sitio característico del paisaje del Centro Histórico de Zacatecas. Reforzando lo anterior, en una de las preguntas de la encuesta se le pidió al participante que eligiera, según su percepción, la imagen más representativa del centro histórico entre un grupo de tres. La asignada con la letra B fue la mayor seleccionada, con un 45.3\%, pues consideraron al edificio catedralicio como hito de la historia y el patrimonio de la ciudad (Ver Figura 6).

Figura 6. La imagen representativa del Centro Histórico conforme a la percepción de los encuestados

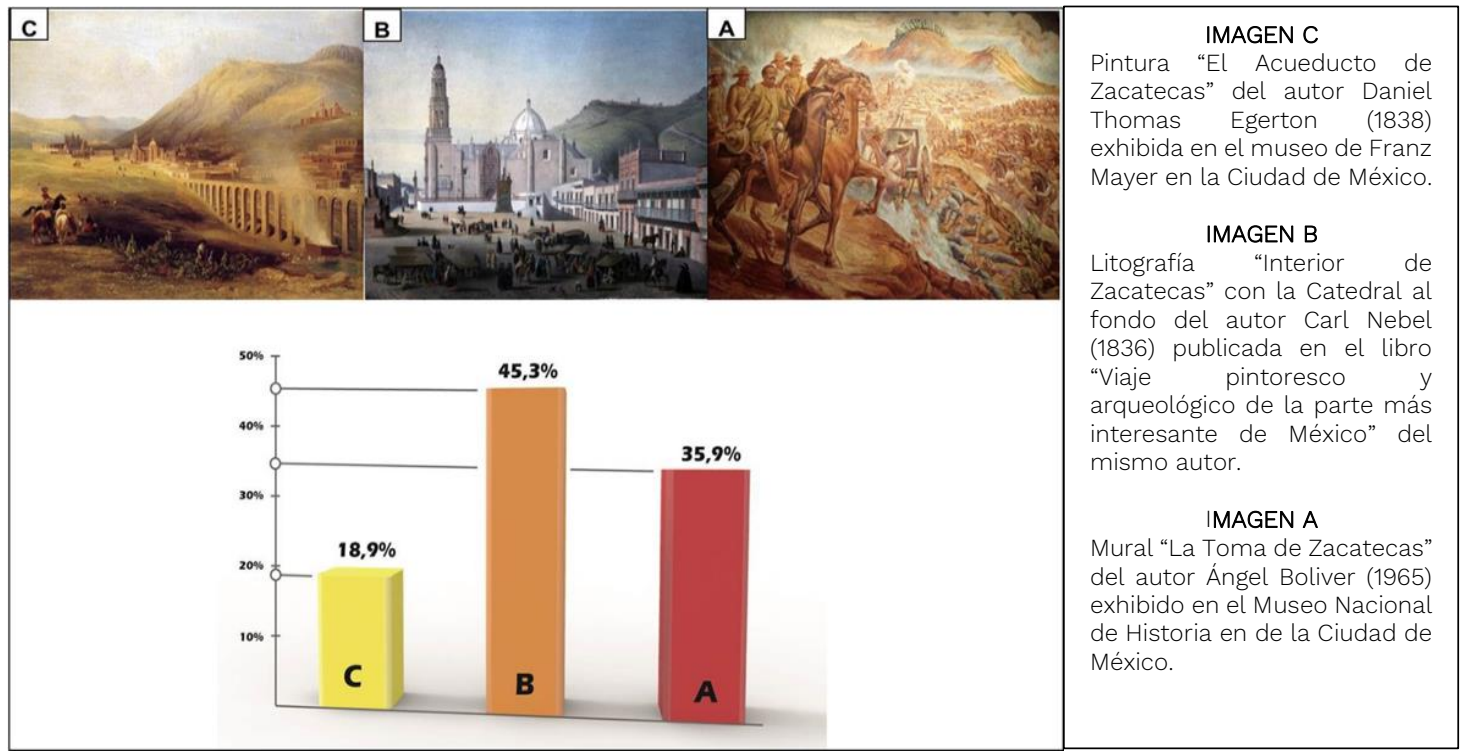

Fuente: Elaboración propia con base en los datos obtenidos en encuestas.

Por el contrario, el componente cultural menos reconocible fue el relacionado con los eventos tradicionales celebrados anualmente. El 58\% de los visitantes no conocen ninguna festividad que se desarrolle en el Centro Histórico de Zacatecas.

Para los componentes naturales del paisaje más conocidos por los encuestados fueron el Cerro de la Bufa y la Mina del Edén. Predomina el Cerro de la Bufa por considerarse un hito natural desde que

ACE, 16 (4.6) CC BY-ND 3.0 ES | UPC Barcelona, España | Percepciones socioculturales del paisaje simbólico: el 12 Centro Histórico de Zacatecas, México. DOI: http://dx.doi.org/10.5821/ace.16.46.9250 
se descubrió su riqueza en minerales que favoreció la consolidación de la ciudad virreinal. Ejemplo de ello es que, para los encuestados, la Batalla de Zacatecas y el Cerro de la Bufa son el evento y el lugar que le dieron su carácter histórico a la ciudad. (Ver Figura 7).

Figura 7. Elección de evento histórico representativo del Centro Histórico conforme a la percepción de los encuestados

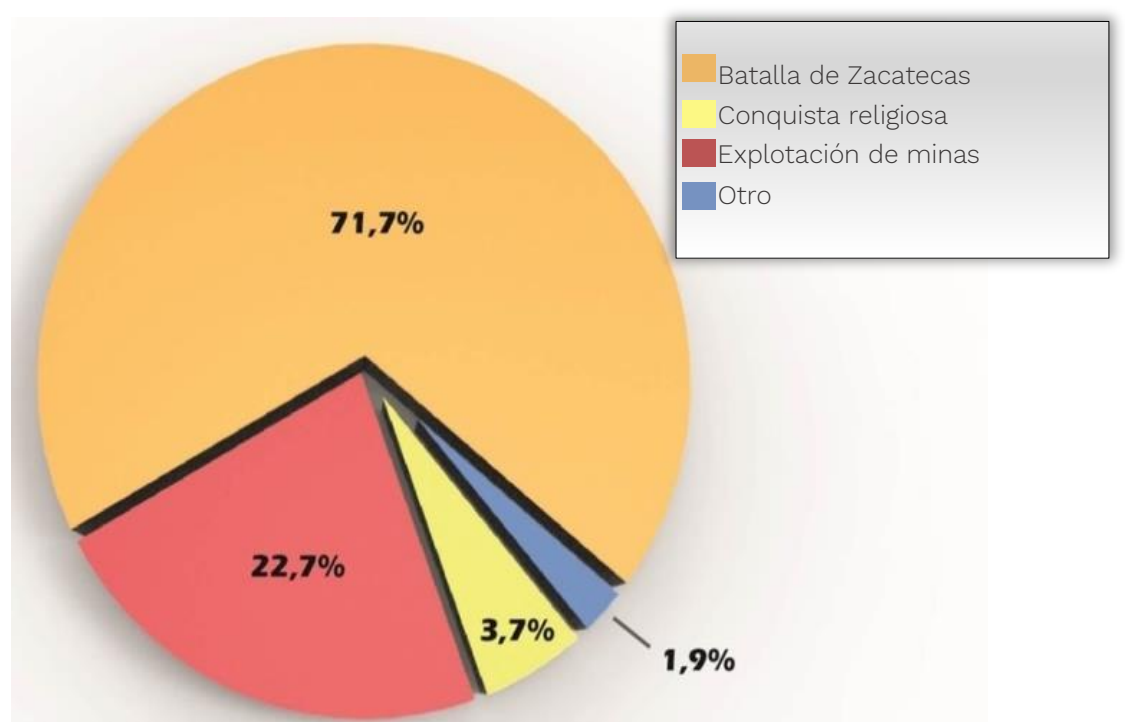

Fuente: Elaboración propia con base en los datos obtenidos en encuestas.

\section{Conclusiones}

El porcentaje mayor de los encuestados fue de $44.3 \%$ dentro del rango de edad de 20 a 40 años, le siguieron los de 40 a 60 años con un $37.7 \%$. Dentro de estas condiciones de edad, el análisis arrojó que le asignan un mayor grado de importancia simbólica a los monumentos y sitios con características históricas, a diferencia de las festividades y los espacios públicos al reconocer a la Catedral como el elemento más significativo de la ciudad. Fue relevante obtener un porcentaje mayor del 90\% en respuestas favorables para la conservación de los hitos más representativos del paisaje. Con el análisis de estos datos, se determinó que los referentes simbólicos del paisaje coinciden en cada uno de los rangos de edad y en los sectores poblacionales, estos referentes formaron parte de sus propias experiencias y cotidianidad. En conclusión, el paisaje simbólico para los actores del Centro Histórico de Zacatecas es el reflejo de sus propias experiencias, donde los hitos culturales y naturalesLa Catedral y el Cerro de la Bufa-formaron parte de sus vínculos territoriales. La valoración de los componentes paisajísticos, según su percepción, estuvo influenciada por las actividades de turismo cultural, una de las más predominantes en Centro Histórico de Zacatecas. La mayoría de los encuestados reconocieron los componentes paisajísticos con mayor riqueza histórica. El componente natural del paisaje el Cerro de la Bufa, fue el hallazgo más significativo de esta investigación, al mostrarse a través de las respuestas obtenidas, que desde su descubrimiento (en el siglo XVI por exploradores españoles en la búsqueda de metales preciosos y después por los fundadores de la ciudad), continúa siendo un componente paisajístico simbólico en el imaginario colectivo, tanto para los visitantes y habitantes del Centro Histórico de Zacatecas. Este componte ha estado ligado con los acontecimientos históricos de la ciudad, como la Batalla de Zacatecas y en la construcción de su propia identidad a través de la imagen simbólica que representa. Se demostró que los hitos naturales, como lo es este relieve, forman parte de los elementos representativos del territorio y que han descrito el paisaje simbólico de las ciudades por sus propios actores.

ACE, 16 (4.6) CC BY-ND 3.0 ES | UPC Barcelona, España | Percepciones socioculturales del paisaje simbólico: el 13 Centro Histórico de Zacatecas, México. DOI: http://dx.doi.org/10.5821/ace.16.46.9250 
El componente cultural del paisaje la Catedral formó parte también de los hallazgos propios de la investigación, al mostrarse las imágenes características del paisaje histórico a los encuestados donde se enmarcaban los eventos e hitos del paisaje como el de la Batalla de Zacatecas. Fue evidente que la mayoría eligió la escena de las actividades en el espacio urbano (letra B de la figura 7), donde coinciden los sentidos y las experiencias diarias en el centro histórico, se identificaron con esta imagen por mostrar un paisaje de afinidad social de acuerdo con las actividades, desde comerciales, culturales, turísticas y hasta religiosas, donde finalmente se encuadra lo simbólico del paisaje a través de la Catedral. Este hito paisajístico ha funcionado como el punto de referencia tanto para visitantes y habitantes, al describir a través de su arquitectura, método constructivo, estilo y materiales a su tiempo, y que la propia población fue construyendo desde siempre como el simbolismo de su ciudad. Entre la Zona $A, B$ y $C$ que corresponden a los perímetros de protección del Centro Histórico de Zacatecas, existe una rotura, al no ser vistos como un todo y partes integrales del entorno paisajístico, en el sentido que existen otros componentes culturales del paisaje que no fueron reconocidos por los encuestados, pero que tienen un valor histórico importante y que bien valdría la pena dar un nuevo enfoque sobre ellos y darlos a conocer a la población habitante y visitante.

El paisaje simbólico es en definitiva la vía para reconocer la imagen de la ciudad percibida que ha trascendido generaciones junto con sus valores histórico-patrimoniales y sus hitos más característicos. Esta investigación contribuyó en mostrar la importancia de los componentes paisajísticos para el reconocimiento e imagen de la ciudad asignándole su propia representatividad paisajística. El paisaje simbólico de Zacatecas está completamente articulado con el valor histórico del Cerro de la Bufa y la Catedral, fue importante considerar este vínculo como parte de su propia imagen e identidad, y no se podría analizar este este territorio sin considerar los valores históricos de estos dos hitos.

Como alcances y otras posibles aportaciones, este análisis podrá ser complementado al integrar otros componentes paisajísticos y su relación con los instrumentos de planeación urbana, para manifestar y proponer estrategias de conservación. También al considerar otras condiciones que influyen en la percepción distintas a las tres analizadas en esta investigación. Para futuras investigaciones será conveniente ampliar el tamaño de la muestra y número de encestas aplicadas para confrontar con los datos obtenidos en este estudio. Finalmente, este artículo ofrece una propuesta de modelo de análisis para otras investigaciones que tengan relación con la percepción del paisaje desde sus componentes simbólicos y para ciudades que contengan características semejantes a las del Centro Histórico de Zacatecas.

\section{Agradecimientos}

Los autores agradecen a la comisión evaluadora por coadyuvar en el perfeccionamiento de la presente publicación.

\section{Autoría}

La autora principal ha compartido y analizado los resultados de la tesis doctoral a través de la elaboración del presente trabajo; el segundo autor aportó algunas ideas puntuales sobre la importancia de conservar los centros históricos y el tercer autor apoyó para el perfeccionamiento del diseño de encuestas y del tamaño de muestras.

Conflicto de intereses: Los autores declaran que no hay conflicto de intereses. 


\section{Bibliografía}

Bailly, A. (1978). La percepción del espacio urbano: Conceptos, métodos de estudio y su utilización en la investigación urbanística. Madrid, España: Instituto de Estudios de Administración Local.

Cárdenas Cruz, J. y Parga Pérez, J. (1991). Monografía geológico-minera del Estado de Zacatecas. Ciudad de México, México: Consejo de Recursos Minerales y Subsecretaría de Minas e Industria Básica.

Censos Económicos INEGI (2014). Calculadora censal de los Censos Económicos. Recuperado de https://www.inegi.org.mx/app/calcen/default.html?p=2004

Cortés Rocha, X. (2014). Planeación participativa en centros históricos: tres casos de estudio: Campeche, Guanajuato y Zacatecas. Ciudad de México, México: PUEC-UNAM.

Edney, J. (1976). Comment on Functional Properties. Environment and Behavior, 8(1), 31-47. DOI: https://doi.org/10.1177/001391657600800103

Domene Verdú, J. F. (2015). Las fiestas de moros y cristianos. Alicante, España: Publicaciones Universidad Alicante.

Flores Olague, J., de Vega, M., Kuntz Ficker, S. y del Alizal, L. (2011). Zacatecas Historia Breve. Ciudad de México, México: Fondo de Cultura Económica.

García Sandoval, B. M. (2014). Las morismas de Bracho en Zacatecas y la cofradía de San Juan Bautista en el siglo XIX (Tesis doctoral). Universidad Autónoma de Zacatecas, Zacatecas, México.

Gobierno del Estado de Zacatecas (2016). Programa Parcial del Centro Histórico de Zacatecas 20162040. Recuperado de http://www.observatorio.gobiernoabiertozacatecas.org.mx/wpcontent/uploads/2017/05/PPCH-Zacatecas-2016-2040-N.pdf

Gobierno del Estado de Zacateas (2018). Leyes, Manuales y Formatos. Gobierno del Estado de Zacatecas 2016 - 2021. Recuperado de https://monumentos.zacatecas.gob.mx/leyes-y-manuales/

Holl, S. (2018). Cuestiones de percepción fenomenología de la arquitectura. Barcelona, España: GG. INEGI (Instituto Nacional de Estadística y Geografía) (2017). Anuario estadístico y geográfico de Zacatecas 2017. SECTUR. Recuperado de https://www.datatur.sectur.gob.mx/ITxEF_Docs/ZAC_ANUARIO_PDF.pdf

INEGI (Instituto Nacional de Estadística y Geografía) (2020). Mapas. Recuperado de https://www.inegi.org.mx/app/mapas//

Manzini Marchesi, L. (2017). Las viviendas de la extraterritorialidad y su influencia en el paisaje cultural vitivinícola del Área Metropolitana de Mendoza. ACE: Arquitectura, Ciudad y Entorno, 12(35), 71-102, DOI: https://doi.org/10.5821/ace.12.35.4689

Nogué, J. (2017). La construcción social del paisaje. Madrid, España: Biblioteca Nueva.

Oviedo, G. (2004). La definición del concepto de percepción en psicología con base en la teoría Gestalt. Revista de Estudios Sociales, (18), 89-96. Recuperado de http://www.redalyc.org/articulo.oa?id=81501809

ACE, 16 (4.6) CC BY-ND 3.0 ES | UPC Barcelona, España | Percepciones socioculturales del paisaje simbólico: el Centro Histórico de Zacatecas, México. DOI: http://dx.doi.org/10.5821/ace.16.46.9250 
Pallasmaa, J. (2018). Los ojos de la piel la arquitectura y los sentidos. Barcelona, España: GG.

Perez-Verdin, G., Lee, M. y Chávez, D. (2004). Outdoor Recreation in a Protected Area in Southern Durango, Mexico: Analysis of Local Residents. Taylor \& Francis, London, UK, Society \& Natural Resources. An International Journal, 17:10, 897-910, DOI: https://doi.org/10.1080/08941920490505310

Rea, L. y Parker, R. (2014). Designing and conducting survey research: A comprehensive guide (Fourth Ed.). San Francisco, USA: Jossey-Bass, Wiley.

Robles Delgado Romero, A. (2017). Una mirada sobre los territorios de transición en las ciudades contemporáneas. Paisajes híbridos del Área Metropolitana de Guadalajara. ACE: Arquitectura, Ciudad y Entorno, 12(35), 103-120. DOI: https://doi.org/10.5821/ace.12.35.4772

Smith, Curtis (1984). The relationship between the pleasingness of landmarks and the judgement of distance in cognitive maps. Journal of Environmental Psychology, (4), 229-234, DOI: https://doi.org/10.1016/S0272-4944(84)80044-4

Stefani, D. (2005). Teoría de la Acción Razonada: Una propuesta de evaluación cuali-cuantitativa de las creencias acerca de la institucionalización geriátrica. Revista Evaluar, 5(1), 22-37. DOI: https://doi.org/10.35670/1667-4545.v5.n1.538

UNESCO (United Nations Educational, S. a.) (1993). Advisory Body Evaluation (ICOMOS) from Historic Centre of Zacatecas. Recuperado de https://whc.unesco.org/en/list/676/documents/

UNESCO (United Nations Educational, S. a.) (2004). World Heritage Convention. The Criteria for Selection. Recuperado de http://whc.unesco.org/en/criteria/

Vergara Durán, A. (2006). Transformaciones de la imagen de una ciudad: Repercusiones de la renovación urbana. Memorias: revista digital de historia y arqueología desde El Caribe (6). Recuperado de https://dialnet.unirioja.es/servlet/articulo?codigo=2209719

Vértiz, J. y Alfaro A. (2001). Moros y cristianos una batalla cósmica. Ciudad de México, México: Artes de México.

Zárate Martín, M. (2016). Paisajes culturales a través de casos en España y América. Madrid, España: UNED.

ACE, 16 (46) CC BY-ND 3.0 ES | UPC Barcelona, España | Percepciones socioculturales del paisaje simbólico: el 